\title{
Towards the regulation of Research Ethics Boards
}

\author{
Joan C. Bevan MD FRCA
}

B URIED in the two-inch thick Thompson report of the Committee of Enquiry on the case involving Dr. Nancy Olivieri, the Hospital for Sick Children, the University of Toronto ( $\mathrm{U}$ of $\mathrm{T}$ ) and Apotex Inc., published on October 20,2001, are two pages of recommendations that could have far-reaching consequences for Research Ethics Boards (REBs) in Canada. ${ }^{1}$ These propose a very broad interpretation of their present mandate by requiring that REBs review all associated research contracts and agreements for industry-sponsored clinical trials to ensure that there is no restriction on disclosure of risks to patients, whether due to safety, or efficacy concerns about the treatment. They specify that REB have the responsibility to ensure that:

- Contracts or agreements do not prevent an investigator from informing subjects, REB, physicians, or the scientific community of risks to participants identified during a trial;

- Sponsors and investigators understand and comply with ethical guidelines set out in the Tri-Council Policy Statement (TCPS) and other norms of research ethics;

- Protocols are consistent with the wording of contracts or agreements, and consent forms are explicit that participants will be informed of any unforeseen risks;

- Reviews of financial and budget matters are conducted to eliminate actual or potential conflicts of interest (delegation to the institution's office of research services is acceptable as long as the REB audits sample protocols annually to ensure consistency);

- Patient care is never compromised and is unaffected by early discontinuation of the trial;

- Institutional policies on access to patient records for research purposes is in compliance with the TCPS guidelines.
The need to state such recommendations implies that the current review process is inadequate. It cannot be assumed that approval of a protocol by an REB proves that investigators and institutions adhere to ethical, scientific and legal guidelines in clinical trials. Have REBs been so ineffective, even negligent, in fulfilling their mandate to protect human research subjects, that regulatory changes are needed?

The problem is that a few research subjects have been harmed during recent clinical trials and limitations in the ethics review process have been blamed. Following the death of a volunteer in an asthma study all research at Johns Hopkins University was suspended "because the ethics committee that approved the study in question had failed to take proper precautions to protect its subjects". ${ }^{2}$ One of the conditions for reinstatement was that all protocols must be reviewed at a meeting of the full board. In the investigation of Jesse Gelsinger's death in a gene therapy trial at the University of Pennsylvania Institute for Human Gene Therapy it was found that "the ethics committee had given too much weight to ensuring informed consent and not enough attention to minimizing the harm associated with participation in research". ${ }^{3}$ Non-disclosure of financial ties between investigators and sponsors led to the suspension of all trials at that institution. After two children were brain damaged in a study which exposed them to lead paint in Baltimore houses, law suits were filed against Kennedy Krieger Institute, affiliated with Johns Hopkins University. The court criticized the ethical review process because "the families were deceived about the inherent dangers". ${ }^{4}$ Implicating the role of the REBs in these tragic cases is sobering.

In the widely publicized case of Canadian physician Dr. Nancy Olivieri, complications and failures of treatment appeared early in a trial of deferiprone in patients with thalassemia. But she had signed a contract in 1995 with Apotex Inc., the trial sponsor, agreeing that “......all information obtained or generated by you during the contract and for a period of one year thereafter,

From the Department of Anaesthesia, University of Toronto, Sunnybrook \& Women's College Health Sciences Centre, Toronto, Ontario, Canada.

Address correspondence to: Dr. Joan C. Bevan, Department of Anaesthesia, Room 203, Women's College Campus, Sunnybrook and Women's Health Sciences Centre, 76 Grenville Street, Toronto, Ontario M5S 1B2, Canada. Phone: 416-323-6008; Fax: 416-323-6307; E-mail: joan.bevan@utoronto.ca 
shall be and remain secret...not to be disclosed...except with the consent of Apotex..." In breach of this clause, Dr. Olivieri published her findings in 1998, in the New England Journal of Medicine. This prompted U of $\mathrm{T}$ to commission Dr. Arnold Naimark to review the case. His report exposed conflicts of interest and recommended harmonization of $\mathrm{U}$ of $\mathrm{T}$ research policies to enforce ethical guidelines and public accountability in research. ${ }^{5}$ Subsequent controversy, legal and disciplinary actions, were sufficiently bizarre to be incorporated into the plot for John le Carré's novel, 'The Constant Gardener'. Dr. Olivieri's eventual vindication by the Thompson report was accompanied by exposure of flaws in the REB system that prompted these seminal recommendations to strengthen the protection of human subjects in clinical research. ${ }^{1}$

Institutional REBs in Canada have been empowered to refuse to give approval, or to withdraw approval from a study for over 25 years, but may lack the infrastructure to meet these new expectations. The unprecedented growth in the volume and complexity of medical research has already taxed the capabilities of these committees and opinions have been expressed that there is a sense of crisis in the overburdened REB system. ${ }^{6}$ REBs depend largely on part-time volunteers, who are unlikely to have either the time or expertise to examine contracts carefully and provide monitoring of study progress, lending support for independent data monitoring and safety committees. ${ }^{7}$ In the United States, clinical research is subject to federal legislation under the Common Rule (1991). ${ }^{8}$ Administration is by the Office for Human Research Protection for clinical research and the Federal Drug Administration for new drug trials. They can, and do, take regulatory actions against medical centres when research ethics are found to be compromised. ${ }^{9}$

Review of research by REBs has not been specifically covered by federal legislation in Canada, but government granting agencies make approval a condition of funding. The Tri-Council Policy Statement $(1998)^{10}$ has set the standard for harmonization of clinical research ethical and scientific reviews by REBs. However, these guidelines do not always give sufficient direction or address many of the rapidly changing ethical concerns that confront REBs. Research reviews meet major ethical challenges in four areas: facilitation of the translation of scientific advances into clinical care, cross disciplinary and multi-centre studies, intellectual property rights and commercialization of research, and harmonizing review standards nationally and internationally. It is not surprising that REBs in Canada may not be able to consistently meet their mandate to protect human subjects. Furthermore, in at least one case the application of the Tri-Council guidelines to a decision on the approval of a new drug trial brought the REB in conflict with the therapeutic products directorate (TPD). ${ }^{11}$ The TPD, the drug licensing board in Canada, endorses International Conference on Harmonization Good Clinical Practice (ICH-GCP) guidelines ${ }^{12}$ which differ from the Tri-Council guidelines in attitudes to placebo-controlled trials.

The decentralized approach to governance of research involving human subjects in Canada has produced a multiplicity of REB structures, without public accountability or transparency. This results in variable standards of ethical review. The funding agencies and Canadian Institutes of Health Research (CIHR) enact guidelines which have no formal enforcement mechanisms. The Thompson report ${ }^{1}$ proposed that "Health Canada should review the current regulations on health research and make appropriate changes to protect the public interest and the rights of patients who volunteer to be subjects of research". Alternative regulatory models need to be considered. In France and Denmark, government legislation provides a clear regulatory framework for REBs, but they lose flexibility at the local and institutional level. The United States system has a formal legal requirement of accountability which allows self-regulation at the institutional level and a publicly accountable system for protecting the rights and welfare of human subjects. In Europe, framework legislation integrates guidelines such as the ICH-GCP, but local implementation is necessary and local regulations may not conform with the framework regulations.

The spectre of potential litigation against REBs and individual responsibility for decisions is now troubling REB members. They question whether the host institution will assume responsibility for their committees' decisions or whether there is likely to be personal liability. Unless REBs are protected by an organizational structure, few will be prepared to risk approving a protocol which puts subjects at any risk: early phase I trials in healthy volunteers will not be approved and innovative drugs will remain untested. Placebo-controlled studies will prevent trials of new treatments for common diseases if standard guidelines are not agreed upon, and genetic therapies may be thwarted because of uncertainty about privacy and confidentiality issues in the use of genetic information. Consequently, it may be more important to improve the standards of the review than to change the system. Professionalization of REBs through licensing and accreditation mechanisms would require formal mandates to be developed which include educational expectations for committee members to ensure scien- 
tific, ethical, legal and patient advocacy representation. Terms of appointment may need to be limited and more external members included. Administrative support for committee work is now commonly provided and consideration for increased budget to meet salaries of members is necessary.

Some initiatives are beginning to address the recommendations of the Thompson report for new standards for REBs. At $\mathrm{U}$ of $\mathrm{T}$, a standard form of contract for academia-industry trials was instituted in April 2001 and the first six months' experiences suggest that voluntary compliance has been successful. ${ }^{13}$ This contract harmonizes the standards across all of the affiliated hospitals and sets principles to ensure the independence and integrity of the research. In conjunction with guidelines for the declaration of scientific independence of investigators from sponsors, now required by editors of most major medical journals before acceptance of research reports for publication, ${ }^{14}$ many of the tensions in the industry-sponsored studies have been addressed. Other areas of concern are looming for REBs. Should REBs undertake trial monitoring or delegate this to independent safety and data monitoring boards? Should clinical trials be registered? Would post research monitoring and meta analysis of results assure the quality of research approved? Is harmonization going to be effective in standardizing the review process?

The mandate of REBs remains unchanged: to protect human subjects in research from harm and to maintain public trust in the clinical research process for the advancement of medical knowledge and clinical care. Present concerns reflect organizational and governance problems for research ethical review in Canada. Reliance on local bureaucratic REB mechanisms without a central, national approach to ethical standardization, and the lack of regulatory enforcement, is no longer workable. Although government intervention and funding will be needed to position REBs in an organizational framework, there are concerns that continuing to depend on voluntariness and institutional support will give government granting agencies even greater control of research governance. Some are concerned about government objectivity because CIHR has already made partnerships with industry, through the Centres of Excellence program, to fund and administer grants to investigate topics of mutual interest. Arguments that the established system can be adapted to meet current needs are based on the belief that flexibility allows more rapid response to changes and accommodates regional needs. Legal empowerment has the disadvantage of rigidity and cannot rapidly meet changing ethical standards.
But, accountability and transparency will only be achieved in a formal regulatory environment in which the government effects changes to the governance of clinical research in Canada. There is a clear need for the introduction of central regulation, in support of an updated REB system which retains local responsibility, to facilitate local, national and international standardization of ethical research review. Otherwise, the impact on research programs of major academic centres because of public distrust of research ethical reviews, could be potentially devastating to promising therapeutic research into new drugs and genetic therapies.

\section{Acknowledgements}

No funding support to acknowledge. This article was written during the completion of a degree in bioethics at the University of Toronto and was inspired by the courses supervised by Dr. Peter Singer and Mr. Trudo Lemmens.

\section{Pour une réglementation des Comités d'éthique de la recherche}

Noyées dans le volumineux rapport Thompson du Comité d'enquête sur le cas impliquant le Dr Nancy Olivieri, l'Hospital for Sick Children, l'University of Toronto (U de T) et Apotex Inc., publié le 20 octobre 2001, se trouvent deux pages de recommandations qui pourraient avoir d'importantes conséquences sur les Comités d'éthique de la recherche (CER) au Canada. ${ }^{1}$ Elles proposent une très large interprétation de leur mandat actuel en réclamant que les CER examinent tous les contrats de recherche en association et les accords conclus pour les études cliniques commanditées par l'industrie afin de s'assurer qu'il n'y a aucune restriction à la divulgation des risques au patient, qu'il s'agisse de la sécurité ou de l'efficacité du traitement. Elles spécifient que les CER ont la responsabilité de s'assurer que :

- Les contrats ou les accords n'empêchent pas un chercheur d'informer les sujets, le CER, les médecins ou les scientifiques à propos des risques découverts en cours d'étude.

- Les commanditaires et les chercheurs comprennent le guide d'éthique mis au point dans 
l'Énoncé de politique des trois conseils (EPTC) et les autres normes déontologiques de la recherche, et s'y soumettent.

- Les protocoles sont conformes aux termes des contrats ou des accords et les formulaires de consentement portent la mention explicite que les participants seront informés de tout risque imprévu.

- L'examen des questions financières et budgétaires est réalisé de manière à éliminer les conflits d'intérêts actuels ou potentiels. La délégation de pouvoirs à la direction du service de la recherche d'un établissement est acceptable à condition que le CER vérifie les protocoles annuellement dans un but de cohérence.

- Le traitement du patient n'est jamais compromis et n'est pas affecté par l'arrêt de l'essai.

- Les politiques institutionnelles sur l'accès au dossier du patient pour des fins de recherche sont compatibles avec les directives de l'EPTC.

La nécessité d'énoncer de telles recommandations laisse entendre que le processus de révision actuel est inadéquat. L'approbation d'un protocole par un CER ne prouve pas que les chercheurs ont suivi les directives éthiques, scientifiques et légales en matière d'essais cliniques. Les CER ont-ils été à ce point inefficaces, voire négligents, en remplissant leur mandat de protéger les sujets humains en recherche, pour que des modifications réglementaires soient nécessaires?

Le fait est que peu de sujets ont subi des effets nuisibles au cours des récentes études cliniques et les limites des révisions déontologiques ont été mises en cause. À la suite du décès d'un volontaire pendant une étude sur l'asthme, toutes les recherches ont été suspendues à la Johns Hopkins University "parce que le comité de révision déontologique qui a approuvé l'étude en question n'a pas pris toutes les précautions pour protéger les sujets". ${ }^{2}$ Une des conditions de la remise en vigueur des études était que tous les protocoles soient révisés par le comité au complet. Dans l'enquête sur le décès de Jesse Gelsinger, au cours d'un essai sur la thérapie génique au University of Pennsylvania Institute for Human Gene Therapy, on a jugé que "le comité de déontologie avait accordé trop de poids au consentement éclairé et pas assez à la réduction des inconvénients associés à la participation à la recherche." ${ }^{3} \mathrm{La}$ non-divulgation des liens financiers entre les chercheurs et les commanditaires a mené à la suspension de tous les essais à cet établissement. Après que deux enfants ont subi des dommages cérébraux pendant une étude qui les exposaient à la peinture au plomb dans des maisons de Baltimore, des poursuites ont été engagées contre le Kennedy Krieger Institute, affilié à la Johns Hopkins University. Le tribunal a blâmé le processus de révision déontologique parce que "les familles ont été trompées quant aux dangers inhérents". 4 Englober le rôle des CER dans ces tragédies donne à réfléchir.

Dans le cas largement diffusé d'un médecin canadien, le Dr Nancy Olivieri, les complications et les défaillances du traitement sont apparues tôt pendant un essai sur le défériprone chez des patients atteints de thalassémie. Par ailleurs, le Dr Olivieri avait signé un contrat en 1995 avec le commanditaire Apotex Inc. acceptant "......de garder secrètes toutes les informations obtenues ou générées par vous pendant la durée du contrat et pour un an après la fin de l'étude...de ne pas les divulguer...sans le consentement d'Apotex..." En violation de cette clause, elle a publié ses résultats en 1998 dans le New England Journal of Medicine. Pressée par la situation, l'U de $\mathrm{T}$ a confié la révision du cas au Dr Arnold Naimark. Son rapport a exposé les conflits d'intérêts et recommandé l'harmonisation des politiques de recherche de l'université concernant l'application du guide d'éthique et l'obligation de rendre compte en matière de recherche. ${ }^{5}$ La controverse qui a suivi, de même que les actions judiciaires et disciplinaires, ont été suffisamment singulières pour servir l'intrigue d'un roman de John le Carré, The Constant Gardener (La constance du jardinier). La défense ultérieure du Dr Olivieri par le rapport Thompson était accompagnée de la révélation de failles dans le système de CER, ce qui a donné lieu à des recommandations substantielles pour renforcer la protection des sujets humains en recherche clinique. $^{1}$

Depuis plus de 25 ans, les CER institutionnels du Canada se sont sentis habilités à approuver ou non une étude, mais ils n'ont peut-être pas l'infrastructure nécessaire pour répondre à ces nouvelles attentes. La croissance sans précédent de la quantité et de la complexité de la recherche médicale a déjà mis à l'épreuve les capacités de ces comités et, selon des opinions émises, il y a un sentiment de crise dans le système surchargé du CER. ${ }^{6}$ Les CER dépendent largement de volontaires à temps partiel, qui ne semblent pas avoir le temps ni les connaissances suffisantes pour vérifier attentivement les contrats et surveiller l'évolution des études, l'assistance pour le contrôle de données indépendantes et les comités de sécurité. ${ }^{7}$ Aux ÉtatsUnis, la recherche clinique est soumise à la législation fédérale sous la Common Rule (1991). ${ }^{8}$ L'administration est réalisée par l'Office for Human Research Protection for clinical research et la Federal Drug Administration pour les essais sur les nouveaux 
médicaments. Ils ont le pouvoir, et l'exercent, puisqu'ils appliquent la réglementation contre des centres médicaux quand ils jugent qu'il y a infraction au code déontologique de la recherche. ${ }^{9}$

Au Canada, l'examen de la recherche par les CER ne fait pas l'objet d'une législation fédérale spécifique, mais les agences de subventions publiques font de l'approbation de l'étude une condition au financement. L'Énoncé politique des trois conseils $(1998)^{10} \mathrm{a}$ établi les normes d'harmonisation de l'éthique en recherche clinique et de la révision scientifique par les CER. Cependant, ces directives ne fournissent pas toujours les orientations nécessaires ou ne traitent pas des nombreux problèmes éthiques qui évoluent rapidement et qui confrontent les CER. La révision de la recherche rencontre des défis éthiques importants dans quatre domaines : la facilitation de l'application cliniques des découvertes scientifiques, les études interdisciplinaires croisées et multicentriques, les droits de propriété intellectuelle et la commercialisation de la recherche et, finalement, l'harmonisation nationale et internationale des normes de révision. Il n'est pas étonnant que les CER du Canada ne puissent exercer leur mandat de protection des sujets humains de manière constante. De plus, dans un cas au moins, l'application des directives des trois conseils à une décision sur l'approbation de l'étude d'un nouveau médicament a entraîné un conflit entre le CER et la direction des produits thérapeutiques (DPT). ${ }^{11} \mathrm{La}$ DPT, office canadien d'homologation des médicaments, appuie les recommandations de l'International Conference on Harmonization Good Clinical Practice (ICH-GCP) guidelines ${ }^{12}$ qui diffèrent de celles des trois conseils quant à l'attitude à adopter dans les essais contre placebo.

L'approche décentralisée de la gouvernance en recherche sur des sujets humains au Canada a provoqué une multiplicité de structures de CER, sans obligation de rendre compte ou de transparence. Il en résulte des normes variables de révision déontologique. Les organismes de financement et les Instituts de recherche en santé du Canada (IRSH) publient des directives qui ne présentent pas de mécanismes d'exécution. Le rapport Thompson ${ }^{1}$ dit que "Santé Canada devrait revoir les réglementations actuelles sur la recherche médicale et faire les modifications appropriées pour protéger l'intérêt public et les droits des patients qui s'engagent comme volontaires dans la recherche sur les sujets humains." D'autres régimes de réglementation doivent être envisagés. En France et au Danemark, la législation gouvernementale présente un cadre de réglementation clair pour les CER, mais ces derniers voient leur marge de pouvoir décisionnel réduite au niveau local et institutionnel. Le système des États-Unis possède une prescription juridique de responsabilité formelle qui permet l'autoréglementation au niveau de l'institution et un système de responsabilité publique pour protéger les droits et le bien-être des sujets humains. En Europe, la législation cadre intègre des directives comme celles de l'ICH-GCP, mais la mise en ouvre locale est nécessaire et les règlements locaux ne correspondent pas toujours aux réglementations cadres.

Le spectre de litiges potentiels contre les CER et la responsabilisation individuelle face aux décisions prises inquiètent maintenant les membres des CER. Ils se demandent si l'établissement-hôte doit assumer la responsabilité des décisions de leur comité ou s'il doit y avoir une responsabilité personnelle. À moins que les CER ne soient protégés par une structure organisationnelle, peu de membres seront prêts à risquer l'approbation d'un protocole où les sujets seraient exposés à quelque risque que ce soit : les essais de phase I précoces chez des volontaires en bonne santé ne seront pas approuvés et des médicaments prometteurs ne seront pas testés. Les essais contre placebo vont empêcher les essais de nouvelles thérapies pour des maladies communes si des directives uniformisées ne sont pas convenues et les thérapies génétiques pourraient être contrecarrées à cause de l'incertitude quant à la protection des renseignements personnels et à la confidentialité dans l'emploi d'informations génétiques. En conséquence, il pourrait être plus important d'améliorer les normes de révision que de changer le système. La professionnalisation des CER, par la délivrance de licence et la reconnaissance des titres de compétence, nécessiterait l'établissement de mandats officiels comprenant des exigences pédagogiques pour les membres du comité afin d'assurer la représentation scientifique, éthique, légale et la défense des droits des patients. Il faudrait peut-être limiter la durée du mandat et accepter plus de membres de l'extérieur. Un soutien administratif est maintenant accordé régulièrement aux travaux du comité mais il est nécessaire d'augmenter le budget afin d'attribuer un salaire aux membres.

Certaines actions ont été entreprises pour donner suite aux recommandations du rapport Thompson visant à établir de nouvelles normes pour les CER. À l'U de T, un contrat de forme normalisée pour les essais conjoints université-industrie a été institué en avril 2001 et, après les six premiers mois, l'expérience de l'autodiscipline est un succès. ${ }^{13}$ Ce contrat concilie les normes de tous les hôpitaux affiliés et définit les principes qui assurent l'indépendance et l'intégrité de la recherche. En corrélation avec les recommandations concernant la déclaration de l'indépendance scientifique des 
chercheurs par les commanditaires, maintenant exigée par les directeurs de la majorité des principaux journaux médicaux avant d'accepter de publier des rapports de recherche, ${ }^{14}$ de nombreux sujets de tension dans les études subventionnées par l'industrie ont été traités. D'autres sujets de préoccupation se manifestent pour les CER. Devraient-ils entreprendre la surveillance de l'essai ou déléguer cette tâche à des commissions indépendantes chargées du contrôle des données et de la sécurité? Devrait-on enregistrer les essais cliniques? La surveillance post-recherche et la méta-analyse des résultats sont-elles des garanties de la qualité de la recherche approuvée? L'harmonisation sera-t-elle utile dans la normalisation du processus de révision?

Le mandat des CER demeure inchangé : protéger des effets nuisibles les sujets humains de la recherche et maintenir la confiance du public dans le processus de recherche clinique pour l'avancement des connaissances médicales et des soins cliniques. Les préoccupations actuelles sont le reflet de problèmes d'organisation et de gouvernance concernant la révision déontologique de la recherche au Canada. La confiance accordée aux mécanismes bureaucratiques locaux, sans une approche centrale et nationale de la normalisation éthique, et l'absence d'exécution de la réglementation ne sont plus raisonnables. Bien que l'intervention du gouvernement et sa contribution financière soient nécessaires pour développer les CER dans un cadre organisationnel, on peut se demander si le fait de dépendre du bénévolat et du soutien institutionnel ne donnera pas aux agences de subventions publiques un plus grand contrôle de la gouvernance de la recherche. Certains se préoccupent de l'objectivité gouvernementale, car les IRSC se sont déjà associés à l'industrie, par le Programme des centres d'excellence, à fournir et administrer des subventions de recherche dans des domaines d'intérêts communs. Les arguments voulant que le système établi puisse être adapté afin de répondre aux besoins actuels reposent sur le fait qu'on croit que la flexibilité permet une réaction plus rapide aux changements et tient compte des besoins régionaux. L'habilitation légale présente le désavantage de la rigidité et ne permet pas de s'adapter rapidement aux modifications des normes d'éthique.

Toutefois, la responsabilisation et la transparence ne seront atteints que dans l'environnement d'une réglementation formelle dans laquelle le gouvernement modifie la gouvernance de la recherche clinique au Canada. Il y a un besoin évident d'une nouvelle réglementation centrale pour appuyer un système de CER modernisé qui maintient une responsabilité locale, facilite la normalisation locale, nationale et internationale de l'examen déontologique de la recherche. Autrement, l'impact sur les programmes de recherche des grands centres universitaires, à cause de la méfiance du public face aux révisions déontologiques, pourrait avoir des effets dévastateurs sur des projets de recherche thérapeutique prometteurs.

\section{Remerciements}

Aucun appui financier à déclarer. Le présent article a été rédigé au terme d'études en bioéthique à l'Université de Toronto et a été inspiré par les cours supervisés par le Dr Peter Singer et M. Trudo Lemmens.

\section{References}

1 Thompson J, Baird P, Downie J. Committee of inquiry on the case involving Dr. Nancy Olivieri, the Hospital for Sick Children, the University of Toronto, and Apotex Inc. Section D Recommendations 10-14 www.dal.ca/committeeofinquiry/

2 Kolata G. Media report: U.S. suspends human research at John Hopkins after death. The New York Times July 20, 2001 and Research volunteer death. John Hopkins Medicine, Press releases. http://www.hopkinsmedicine.org/press/index.html

3 Sibbald B. Death but one unintended consequence of gene-therapy trial. CMAJ 2001; 164: 1612.

4 Rioux P. Media report: Baltimore lead abatement study faulted by court. IRED.com, Inc. September 10, 2001 and Kennedy Kreiger Institute, Johns Hopkins response to Court of Appeals ruling in lead paint case. Johns Hopkins Medicine, Press Releases http://www.hopkinsmedicine.org/press/index.html

5 Knoppers BM, Lowy FH, Naimark A. Clinical trials of Ll (Deferiprone) at The Hospital for Sick Children: a review of facts and circumstances. http://www.sickkids.ca/Lltrials/revcontents.asp

6 Levine RJ. Institutional Review Boards: a crisis in confidence (Editorial). Ann Int Med 2001; 134: 161-3.

7 Hampton JR. Clinical trial safety committees: the devil's spoon. BMJ 2000; 320: 244-5.

8 Anonymous. Federal policy for the protection of human subjects ("Common Rule"). Federal Register 1991; 56: 28002 and the Common Rule 1991. Introduction to human subject and privacy protections at the National Institute of Justice. wysiwyg://45/http://www.ojp.usdoj.gov/nij/human subjects/hs_0l.html

9 Burman WJ, Reves RR, Cohn DL, Schooley RT. Breaking the camel's back: multicenter clinical trails and local institutional review boards. Ann Intern Med 2001; 134: 152-7.

10 Tri-Council policy statement: ethical coduct for research involving humans. August 1998. http://www.nserc.ca 
/programs/ethics/english/policy.htm

11 Weijer C. Placebo trials and tribulations. CMAJ 2002;

166: 603-4.

12 Hirtle $M$, Lemmens T, Sprumont D. A comparative analysis of research ethics review mechanisms and the ICH good clinical practice guideline. European Journal of Health Law 2000; 7: 265-92.

13 Naylor CD. Early Toronto experience with new standards for industry-sponsored clinical research: a progress report. CMAJ 2002; 166: 453-6.

14 Anonymous. Look, no strings: publishing industryfunded research (Editorial). CMAJ 2001; 165: 733. 